\title{
Systemic Chemotherapy for Resectable Hepatic Colorectal Metastases: Adjuvant, Neoadjuvant, or Not at All?
}

\author{
Clinton T. Morgan · Clifford S. Cho
}

Published online: 27 March 2014

(C) Springer Science + Business Media New York 2014

\begin{abstract}
The contemporary management of hepatic colorectal adenocarcinoma metastasis relies on the multidisciplinary use of both local operative resection and systemic chemotherapy. This article will discuss the principles and data that are of relevance in determining the optimal way to combine these treatment modalities. In so doing, we will explore the issue of when and whether to administer chemotherapy for patients with resectable hepatic metastases.
\end{abstract}

Keywords Colorectal adenocarcinoma - Liver metastases . Surgery $\cdot$ Systemic chemotherapy $\cdot$ Neoadjuvant $\cdot$ Adjuvant

\section{Introduction}

It is easy to overlook how profoundly surprising the efficacy of hepatic metasectomy actually is for patients with stage IV colorectal adenocarcinoma. That a local therapy could possibly impact the course and outcome of a systemically disseminated cancer is, on some level, counterintuitive. Nevertheless, the integration of local surgical

This article is part of the Topical Collection on Colorectal Liver Metastases.

C. T. Morgan

Department of Surgery, University of Wisconsin School of Medicine and Public Health, Madison, WI, USA

e-mail: cmorgan@uwhealth.org

C. S. Cho $(\square)$

Department of Surgery, Section of Surgical Oncology, University of Wisconsin School of Medicine and Public Health, J4/703 Clinical Sciences Center, 600 Highland Avenue, Madison, WI 53792-7375, USA

e-mail: cho@surgery.wisc.edu metastasectomy and systemic chemotherapy has been well established as the most effective way to treat hepatic colorectal adenocarcinoma metastases. This review will explore the question of how to best navigate the interface between local and systemic therapy. Specifically, it will ask: how do we best utilize chemotherapy for patients with surgically resectable hepatic metastases? In addition to exploring the two most commonly debated options (chemotherapy as neoadjuvant therapy before hepatic resection versus chemotherapy as adjuvant therapy after hepatic resection), we will also consider a third option-avoiding the routine use of chemotherapy in the perioperative period.

\section{The Efficacy of Operative Hepatic Metastasectomy Alone}

The contemporary treatment of metastatic colorectal adenocarcinoma clearly involves a close multidisciplinary coordination of surgical and chemotherapeutic interventions. Given the fact that surgical resection and chemotherapy are so often used together, it is fairly difficult to measure the independent impact that hepatic resection alone imparts on the disease course of metastatic colorectal adenocarcinoma. To do so, it is useful to recall the historical outcomes of patients with metastatic colorectal adenocarcinoma before the availability of effective systemic therapy [1-4]. Reports from that era describe average survival durations of approximately 6 months for patients with unresectable stage IV colorectal adenocarcinoma. Not surprisingly, metastases whose distributions are confined to the liver represent a slightly favorable phenotypic manifestation of disease; indeed, the average survival of untreated patients with liver-only stage IV disease approximated 
1 year. Early reports of surgical therapy administered to carefully selected patients with liver-only metastases in eras of what we would now consider to have been suboptimal chemotherapy described average survival outcomes of approximately 2-3 years.

To identify the purest oncological outcome of a specific treatment intervention, it is helpful to measure not overall survival, which can be affected by many other events and interventions, but recurrence-free survival. House and colleagues reported long-term recurrence-free survival outcomes of 1,037 patients treated with hepatic metastasectomy at the Memorial Sloan-Kettering Cancer Center between 1985 and 1998 [5•]. Treatment in this era (a period dominated by the use of what one could retrospectively argue to have been suboptimal chemotherapy) resulted in recurrence-free survival curves that flattened out at approximately $20 \%$ after a decade-suggesting that approximately one-fifth of carefully selected patients with limited, resectable hepatic metastases can actually be cured of cancer after surgical resection. The biological implications of these data are also reflected in a similar analysis of actual (not actuarial) survivors of hepatic metasectomy reported by Tomlinson and colleagues from the same institution [6॰]. When survival analysis was restricted to 612 patients with long-term follow-up (limiting the analysis to patients who underwent treatment in an era of suboptimal chemotherapy), the actual survival curve again flattened out at about $20 \%$ after 10 years.

\section{The Impact of Chemotherapy}

Contemporary series suggest the strong possibility that survival for patients treated with hepatic metastasectomy are improving. A review of such series (Table 1) indicates that the median survival for patients selected to undergo partial hepatectomy to eradicate their hepatic colorectal adenocarcinoma metastases now approaches $4 \frac{1}{2}$ years [5•, 6•, 7-10]. There is little doubt that improvements in operative technique and perioperative care have had something to do with this trend. However, it is important to recognize that there has also been an equally impressive improvement in the median survival of patients with inoperable stage IV colorectal adenocarcinoma. As systemic chemotherapy has evolved from single-agent 5-fluorouracil (5-FU) to 5-FU/levamisole combinatorial therapy to 5-FU/leucovorin combinatorial therapy to the addition of irinotecan and oxaliplatin and the introduction of so-called biological agents such as bevacizumab and cetuximab, the median survival of patients with even inoperative hepatic colorectal adenocarcinoma metastases has increased from 6 months to about 2 years [11-16]. Clearly, improvements in survival among patients treated
Table 1 Published series of outcomes after hepatic colorectal adenocarcinoma metastsectomy

\begin{tabular}{llrcl}
\hline Author & Era & $n$ & $\begin{array}{l}\text { Operative } \\
\text { mortality } \\
(\%)\end{array}$ & $\begin{array}{l}\text { 5-Year } \\
\text { OS (\%) }\end{array}$ \\
\hline Andreou et al. [10] & $1997-2010$ & 378 & 3 & 53 \\
House et al. [5•] & $1999-2004$ & 563 & 1 & $51^{\text {a }}$ \\
Wei et al. [9] & $1992-2002$ & 423 & 2 & 47 \\
Choti et al. [8] & $1993-1999$ & 133 & $<1$ & 58 \\
House et al. [5•] & $1985-1998$ & 1037 & 3 & $37^{\text {a }}$ \\
Choti et al. [8] & $1984-1992$ & 93 & $<1$ & 31 \\
Scheele et al. [7] & $1960-1992$ & 1718 & 4 & 39 \\
\hline
\end{tabular}

OS overall survival

${ }^{a}$ Five-year disease-specific survival

with surgical metastasectomy have been enabled by improvements in systemic chemotherapy, and there is no debate regarding the importance of using both surgical intervention and systemic therapy in the long-term management of patients with metastatic colorectal adenocarcinoma.

However, justification for the routine use of chemotherapy in the short-term, perioperative period for patients undergoing hepatic metastasectomy is, in truth, not clear. In large measure, reliance on chemotherapy for patients undergoing hepatic metastasectomy is a natural extrapolation of the clear benefits seen with adjuvant chemotherapy after partial colectomy in patients with nodepositive primary colorectal adenocarcinoma. Prospective randomized controlled trials have shown that postoperative chemotherapy-even the use of what would now be considered suboptimal chemotherapy, such as 5-FU/levamisole-reduces the risk of future disease recurrence for patients with resected stage III colon adenocarcinoma [17]. It would seem entirely logical to suppose that, if additional chemotherapy decreases the likelihood of recurrence after primary tumor resection for patients at risk of metastatic dissemination, it would also be helpful in decreasing the likelihood of recurrence after eradication of established metastases as well. What have prospective randomized clinical trials shown us about this potential benefit?

\section{Prospective Randomized Controlled Trial Data (Table 2)}

In 2006, Portier and colleagues published the outcomes of the FFCD ACHBTH AURC 9002 trial [18••]. In this study, which began accrual in 1991, patients undergoing complete 
Table 2 Randomized prospective clinical trials of hepatic metastasectomy with or without systemic chemotherapy

\begin{tabular}{|c|c|c|c|c|c|c|c|}
\hline Author & Era & Arm & $n$ & $\begin{array}{l}5-\text { Year OS } \\
(\%)\end{array}$ & $p(\mathrm{OS})$ & $\begin{array}{l}\text { 5-Year DFS } \\
(\%)\end{array}$ & $p$ (DFS) \\
\hline \multirow[t]{2}{*}{ Portier et al. $[18 \bullet \bullet]$} & \multirow[t]{2}{*}{$1991-2001$} & Resection alone & 100 & 42 & & 27 & \\
\hline & & Resection $\rightarrow$ 5FU/leucovorin & 100 & 51 & 0.13 & 34 & 0.028 \\
\hline \multirow[t]{2}{*}{ Mitry et al. $[19 \bullet \bullet]$} & \multirow[t]{2}{*}{$1991-2001$} & Resection alone & 135 & 40 & & 28 & \\
\hline & & Resection $\rightarrow$ 5FU/leucovorin & 292 & 53 & 0.095 & 37 & 0.058 \\
\hline \multirow[t]{2}{*}{ Ychou et al. [20] } & \multirow[t]{2}{*}{ 2001-2006 } & Resection $\rightarrow$ 5FU/leucovorin & 153 & $72^{\mathrm{a}}$ & & $46^{\mathrm{b}}$ & \\
\hline & & Resection $\rightarrow$ FOLFIRI & 153 & $73^{\mathrm{a}}$ & 0.69 & $51^{\mathrm{b}}$ & 0.44 \\
\hline \multirow{2}{*}{$\begin{array}{l}\text { Nordlinger et al. }[21 \bullet \bullet \\
22 \bullet \bullet]\end{array}$} & \multirow[t]{2}{*}{$2000-2004$} & Resection alone & 182 & 48 & & $30^{\mathrm{c}}$ & \\
\hline & & FOLFOX4 $\rightarrow$ resection $\rightarrow$ FOLFOX4 & 182 & 51 & 0.34 & $38^{\mathrm{c}}$ & 0.068 \\
\hline
\end{tabular}

hepatic metastasectomy (R0 resections) were randomized to receive postoperative 5-FU/leucovorin (the standard chemotherapeutic regimen at the time) or observation alone. Unfortunately, this study was afflicted by poor accrual, and only 173 patients ultimately met all inclusion criteria for analysis by 2001. Thanks in part to this gradual accrual, the study did benefit from prolonged observation, with a median follow-up of approximately 89 months. During that time, no difference in overall survival was observed between the groups $(p=0.13)$. The authors did report a statistically significant difference in disease-free survival $(p=0.028)$, but this had a magnitude of only 6 months.

In 2008, Mitry et al. [19••] reported a combined analysis of this and a separate but similar clinical trial that also met difficulties with patient accrual. This pooled analysis of 302 patients randomized to undergo adjuvant 5-FU/leucovorin chemotherapy or observation following R0 hepatic metastasectomy again identified no difference in overall survival ( $p=0.095$ ) and a 9-month improvement in progression-free survival that did not strictly reach statistical significance $(p=0.058)$. A safe conclusion from these carefully executed efforts is that the oncological impact of postoperative chemotherapy after hepatic colorectal adenocarcinoma metastasectomy is a potential (but moderate) delay in disease progression that ultimately does not affect overall survival.

In retrospect, an obvious problem with these analyses is their use of 5-FU/leucovorin. Although this regimen has been proven to have benefit in the prevention of disease recurrence after removal of primary colorectal adenocarcinoma, it has been surpassed in efficacy by other combinatorial approaches to chemotherapy. It is therefore worth considering a more recent prospective randomized clinical trial reported by Ychou et al. [20]. In this study, which was performed between 2001 and 2006, 306 patients who underwent R0 hepatic metastasectomy were randomized to receive either 5-FU/leucovorin chemotherapy or the more contemporary formulation of FOLFIRI (5-FU/leucovorin/ irinotecan) chemotherapy. Interestingly, despite the incremental advantages of FOLFIRI over 5-FU/leucovorin, there was no difference seen in either overall survival ( $p=0.69)$ or disease-free survival $(p=0.44)$ between the two treatment groups.

The most recent prospective randomized clinical trial evaluating the benefit of chemotherapy for patients undergoing hepatic metastasectomy is the EORTC 40983 trial, the initial results of which were published by Nordlinger et al. [21••]. In this large multicenter trial, 364 patients with one to four hepatic colorectal adenocarcinoma metastases were randomized to undergo hepatic metastectomy with perioperative FOLFOX6 chemotherapy (3 cycles administered prior to resection and 3 cycles administered after resection) or hepatic metastasectomy alone. The study was designed with progression-free survival as its intended endpoint. Following accrual between 2000 and 2004, median follow-up at the time of the study's initial report was 47 months. Patients who were randomized to receive perioperative chemotherapy demonstrated a 7-month improvement in progression-free survival that did not reach statistical significance $(p=0.058)$. At the time of the last reported follow-up (after a median follow-up of 102 months), no differences were observed in overall survival $[22 \bullet \bullet]$.

The results of this trial deserve closer inspection. In order to minimize the impact of inescapable lead time biases arising from the fact that one group underwent operative exploration sooner than the other, the study design considered all patients who developed early disease progression (in the first 20 weeks) or could not undergo 
therapeutic resection to have progressed at week 10 . It is noteworthy that $13 \%$ of patients randomized to receive no perioperative chemotherapy ultimately underwent nontherapeutic operations, whereas only $2 \%$ of patients randomized to receive perioperative chemotherapy underwent non-therapeutic operations. This $11 \%$ difference is reflected by a sharp separation of the Kaplan-Meier progression-free survival curves at the 10-week mark, the magnitude of which is approximately $11 \%$. Interestingly, the magnitude of the separation between the two survival curves does not change over the course of follow-up; after the 10-week mark, the two survival curves are largely parallel. The parallel nature of these survival curves suggests that the differential in progression-free survival was the result of an early event-namely, the ability to deliver a potentially therapeutic resection [23]. It is known that chemotherapy can convert some patients with radiographically unresectable disease to resectability; it is therefore possible that the preoperative administration of chemotherapy in this trial might have allowed a subset of patients to have undergone a resection that might not have been feasible had they gone directly to operative exploration.

One conservative conclusion from these prospective randomized studies can be that the addition of chemotherapy to hepatic metastasectomy may help to delay the onset of disease recurrence in some patients. A second conclusion would have to be that the magnitude of this delay is rather small. A third conclusion, based largely on the EORTC 40983 trial, is that preoperative administration of chemotherapy may provide an added benefit of subtly decreasing the possibility of encountering unresectable disease at the time of operation. A fourth conclusion is that routine administration of chemotherapy before and/or after operative resection does not affect long-term overall survival. Based on these conservative conclusions, most treating physicians and surgeons err on the side of favoring combined modality therapy for patients with resectable hepatic colorectal adenocarcinoma metastases. Indeed, for many, the debate is not whether to give chemotherapy, but when.

\section{Arguments for and Against Neoadjuvant Chemotherapy}

In general, arguments for neoadjuvant chemotherapy follow four lines of reasoning. In no particular order, the first argument for neoadjuvant chemotherapy is to enhance the feasibility of resection. One illustrative example of this can be found in the treatment of locally advanced cases of gastrointestinal stromal tumor (GIST), in which complex and multivisceral resections may be needed to permit therapeutic, margin-negative tumor extirpation. Because of the favorable response rate of GIST to tyrosine kinase inhibition, preoperative imatinib can, on occasion, induce enough tumoral regression to facilitate complete tumor removal [24, 25]. Like GIST, metastatic colorectal adenocarcinoma has become a disease with effective systemic treatment options. Indeed, favorable response rates have motivated the use of preoperative chemotherapy as a means of converting unresectable cases of hepatic metastasis to resectability. However, the extent to which tumoral regression may enhance the feasibility of resection for cases of resectable disease (the focus of the present paper) is unclear. In fact, enthusiasm for preoperative chemotherapy for patients with resectable disease is tempered by the potential for the opposite outcome-heightened risks of hepatic resection resulting from chemotherapy-associated hepatotoxicity. The introduction of newer and more effective chemotherapy has also brought increased risks of side effects that can impede aggressive hepatic resection [26•, 27-33]. Agents such as 5-FU and irinotecan have been associated with hepatic steatosis, which can progress to steatohepatitis and frank hepatic fibrosis. Hepatic steatosis has been linked with risks of intraoperative hemorrhage and postoperative hepatic failure. Oxaliplatin has been associated with the induction of sinusoidal obstruction, which can increase the risk of intraoperative hemorrhage. It appears that these adverse hepatic architectural and functional changes are cumulative; retrospective analyses performed at the MD Anderson Cancer Center suggest that the risk of postoperative hepatic failure doubles from less than $5 \%$ to over $10 \%$ after 9 or more cycles of preoperative chemotherapy. Moreover, so-called biological agents such as bevacizumab appear to potentiate the risk of hepatotoxicity; the risk of postoperative hepatic failure doubles again to over $20 \%$ in patients who have received nine or more cycles of preoperative FOLFOXbevacizumab [26•]. In the EORTC 40983 trial, $13 \%$ of patients in the perioperative chemotherapy arm who did not undergo resection were unable to do so because of hepatic toxicity (compared with $0 \%$ in the no perioperative chemotherapy arm); in addition, the prevalence of postoperative complications was significantly higher in the perioperative chemotherapy arm than in the no perioperative chemotherapy arm (25 vs. $16 \%, p=0.04)$. Prolonged systemic chemotherapy can cause enough deterioration of hepatic function to render patients with radiographically resectable disease unsuitable for operative therapy. Some have advocated for the liberal use of preoperative liver biopsy to assess for hepatotoxicity in patients previously treated with extensive chemotherapy [27].

A second general argument for the routine use of neoadjuvant therapy is that it can eradicate local microscopic disease, thereby enhancing local cancer control. An example of this argument is found in the multimodal 
approach to locally advanced rectal cancer, for which preoperative chemoradiation decreases the likelihood of local recurrence after resection [34]. This general argument is best applied to cancers in which resectability is anatomically confined. In the example of rectal cancer, a surgeon's ability to exert maximal local disease control is limited by the fixed anatomic borders of the bony pelvis. Indeed, assiduous attention to radical resection using total mesorectal excision may mitigate the benefit of preoperative chemoradiation therapy [35]. The extent to which this anatomic confinement affects the conduct of hepatic surgery is less obvious, particularly in the setting of the resectable hepatic colorectal metastasis. The subtle improvement in progression-free survival seen in patients treated with perioperative chemotherapy in the EORTC 40983 trial suggests that local (i.e., intrahepatic) cancer control may be slightly improved with the use of neoadjuvant chemotherapy; however, it is important to note that a similarly subtle improvement in progression-free was seen in the trials evaluating adjuvant chemotherapy as well.

A third argument for neoadjuvant chemotherapy is that it helps to ensure that patients will receive chemotherapy. A practical example of this argument is in the multimodal treatment of gastric cancer, which has been shown to improve outcomes compared with operative resection alone [36, 37]. Because some patients will not recover well enough from gastric resection to then receive postoperative chemotherapy, many patients receive some of their chemotherapy before operation. Despite significant improvements in intraoperative and perioperative care, hepatic resection remains a major operative undertaking associated with a significant risk of morbidity; even at high volume centers, the risks of major complications and prolonged recovery following major hepatic resection remain $20-30 \%[38,39]$. It stands to reason that patients who develop major postoperative complications are at risk of not receiving post-resection chemotherapy. Indeed, in the EORTC 40983 trial, $24 \%$ of patients in the perioperative chemotherapy arm who underwent hepatic metastasectomy did not receive the postoperative phase of their chemotherapy. Therefore, routine administration of chemotherapy prior to surgical intervention may be a means of improving the likelihood of delivering multimodal therapy.

A fourth argument in favor of neoadjuvant chemotherapy is that it permits a window of time during which the kinetics of an individual's disease biology may be measured (as reflected by response to preoperative therapy). This argument is used to support preoperative therapy for resectable pancreatic ductal adenocarcinoma [40]. Because of its aggressiveness, pancreas cancer will occasionally manifest metastatic dissemination during or after preoperative therapy. For this reason, preoperative treatment can enhance patient selection by avoiding non-therapeutic operative interventions. Hepatic metastasectomy is a potentially morbid procedure, so improvement of therapy selection would be useful in the treatment of hepatic colorectal adenocarcinoma metastases. In one analysis from the Memorial Sloan-Kettering Cancer Center, it was observed that disease progression after preoperative chemotherapy was strongly associated with worse outcomes following eventual hepatic metastasectomy [41]. More recent analyses have been mixed, with some series confirming the prognostic significance of tumoral response to chemotherapy, and others studies identifying no prognostic influence [42, 43]. Importantly, surgical intervention is likely to remain the best therapeutic option even for patients whose metastases are unresponsive to chemotherapy; in this regard, the prognostic impact of disease progression on chemotherapy may not be significant enough to contraindicate hepatic metastasectomy.

\section{The Argument for No Perioperative Chemotherapy}

As mentioned earlier, the major randomized clinical trials indicate that postoperative or perioperative administration of chemotherapy may delay the onset of disease recurrence or progression, but does not prolong overall survival. The same conclusions were made in a recent meta-analysis of studies examining perioperative chemotherapy. In general, when an oncological therapy impacts disease-free survival but not overall survival, it implies that the prognostic impact of disease recurrence (or progression) is diluted - either by a disease biology so indolent that recurrence is not particularly dangerous or by the availability of effective salvage therapy [44]. The latter explanation may be relevant for metastatic colorectal adenocarcinoma. When patients develop metastatic disease recurrence or progression, it is very likely that they will eventually be treated with chemotherapy. It is well established that systemic chemotherapy can prolong survival for patients with metastatic colorectal adenocarcinoma. Thus, it may be that the reason clinical trials have never identified an overall survival benefit for chemotherapy may be that later use of chemotherapy was able to "salvage" patients who developed recurrent or progressive disease after surgical resection alone by prolonging their overall survival. This possibility is not without precedent; similar observations have been made regarding the use of adjuvant imatinib following resection of gastrointestinal stromal tumor (GIST) [45].

When viewed in this light, it may be worth asking whether perioperative or adjuvant chemotherapy could be avoided altogether, in effect "saving" chemotherapy in case it should become absolutely necessary (i.e., at the time of postresection disease recurrence). The patients in the aforementioned clinical trials who had been randomized to 
Table 3 Oncological characteristics of patients enrolled in prospective randomized clinical trials of hepatic metastasectomy with or without systemic chemotherapy

a $>24$ months

b $\leq 1$ segment

\begin{tabular}{llc}
\hline & $\begin{array}{l}\text { Adjuvant trials } \\
\text { (Portier et al. }[18 \bullet \bullet ; \\
\text { Mitry et al. } \\
[19 \bullet \bullet])(\%)\end{array}$ & $\begin{array}{l}\text { EORTC 40983 } \\
\text { (Nordlinger et al. } \\
[21 \bullet \bullet])(\%)\end{array}$ \\
\hline Node-negative primary disease & 53 & 45 \\
Disease-free interval $>12$ months & 57 & $27^{\mathrm{a}}$ \\
Single hepatic metastasis & 67 & 51 \\
Hepatic metastases $\leq 5 \mathrm{~cm}$ & 74 & $\sim 50$ \\
Resection of $\leq 2$ hepatic segments & 57 & $45^{\mathrm{b}}$ \\
\hline
\end{tabular}

undergo surgical therapy alone did not appear to suffer adverse outcomes in terms of overall longevity when managed in this manner. One could also ask a more provocative question: is it possible that patients in these trials might have been introduced to subtle, unanticipated risks by virtue of having received perioperative or adjuvant chemotherapy? Non-controlled, retrospective series suggest that when patients treated with hepatic metastasectomy plus chemotherapy recur, they are more likely to develop extrahepatic recurrences than patients initially treated with hepatic metastasectomy alone [46-48]. In the context of retrospective analysis, such associations are likely to be a result of subtle selection biases (i.e., the use of chemotherapy was likely to have been motivated by factors that also made the eventual development of extrahepatic metastasis more likely). But is it possible that the liberal use of chemotherapy in a setting of minimal disease could impact disease biology by selecting out clonal populations of cancer with a more aggressive phenotype? A recent retrospective analysis of patients with hepatic colorectal adenocarcinoma from MD Anderson Cancer Center concluded that patients who had originally received adjuvant FOLFOX after resection of their original stage III primary colorectal cancer did worse after hepatic metastasectomy than patients who, after having had their primary cancers resected, received adjuvant 5-FU alone or no chemotherapy at all (3-year overall survival of 58, 70, and $84 \%$, respectively for patients who received FOLFOX, 5-FU, or no chemotherapy) [49•]. In addition, a significantly higher percentage of KRAS mutations were seen among patients who had received FOLFOX (57 vs. $29 \%$ and $32 \%$, respectively). Although selection bias may have also influenced these associations, these data collectively raise the possibility that overzealous use of chemotherapy may negatively impact the long-term biological course of metastatic colorectal adenocarcinoma in subtle ways.

\section{Conclusions}

Before extrapolating too much from the findings of the aforementioned clinical trials, it is worth examining the clinicopathological variables that characterized the patients enrolled. As outlined in Table 3, a substantial proportion of the patients in the postoperative and perioperative chemotherapy trials had relatively favorable oncological characteristics. Indeed, it could be stated that the patients that populated these landmark analyses do not adequately reflect the typical patient undergoing evaluation for hepatic metastasectomy. When viewed in this light, a more appropriate conclusion to draw from these clinical trials may be that patients with relatively low disease burden or favorable disease characteristics will not derive significant benefit from the use of postoperative or perioperative chemotherapy. Might the survival impact of chemotherapy have been more pronounced if these studies had been limited to patients with higher burdens of metastatic disease? Parks and co-authors from Memorial Sloan-Kettering Cancer Center made the intriguing observation that the magnitude of survival benefit associated with adjuvant chemotherapy was magnified when oncological measures of tumor burden such as the tumor number or Clinical Risk Score were increased [50॰]. In other words, it may therefore be more appropriate to individually tailor the decision of whether to use chemotherapy in addition to hepatic metastasectomy. For patients with limited disease, it may be reasonable to avoid the routine addition of chemotherapy, understanding that chemotherapy may be just as effective when used to treat future recurrences if and when they occur. For patients with extensive disease burden (for whom the likelihood of recurrence is high), a combinatorial approach using perioperative chemotherapy may have a long-term survival benefit. Perhaps future clinical trials should pay less attention to the binary question of whether to use chemotherapy and greater attention to the more nuanced issue of when.

\section{Compliance with Ethics Guidelines}

Conflict of Interest Clinton T. Morgan and Clifford S. Cho declare that they have no conflict of interest.

Human and Animal Rights and Informed Consent This article does not contain any studies with human or animal subjects performed by any of the authors. 


\section{References}

Papers of particular interest, published recently, have been highlighted as:

- Of importance,

-• Of major importance

1. Logan SE, Meier SJ, Ramming KP, Morton DL, Longmire WP Jr. Hepatic resection of metastatic colorectal carcinoma: a ten-year experience. Arch Surg. 1982;117(1):25-8.

2. Wagner JS, Adson MA, van Heerden JA, Adson MH, Ilstrup DM. The natural history of hepatic metastases from colorectal cancer: a comparison with resective treatment. Ann Surg. 1984;199(5):502-7.

3. Cady B, McDermott WV. Major hepatic resection for metachronous metastases from colon cancer. Ann Surg. 1985;201(2):204-9.

4. Petrelli NJ, Nambisan RN, Herrera L, Mittleman A. Hepatic resection for isolated metastasis from colorectal cacinoma. Am J Surg. 1985;149(2):205-9.

5. - House MG, Ito H, Gonen M, et al. Survival after hepatic resection for metastatic colorectal cancer: trends in outcomes for 1,600 patients during two decades at a single institution. J Am Coll Surg. 2010;210(5):744-52. Analysis of survival outcomes following hepatic metastasectomy before and after the availability of effective systemic chemotherapy.

6. - Tomlinson JS, Jarnagin WR, DeMatteo, et al. Actual 10-year survival after resection of colorectal liver metastases defines cure. J Clin Oncol. 2007;25(29):4575-80. Longitudinal follow-up data suggesting that approximately $20 \%$ of patients are actually cured of their metastatic colorectal adenocarcinoma after hepatic metastasectomy.

7. Scheele J, Stang R, Altendorf-Hofmann A, Paul M. Resection of colorectal liver metastases. World J Surg. 1995;19(1):59-71.

8. Choti MA, Sitzmann JV, Tiburi MF, et al. Trends in long-term survival following liver resection for hepatic colorectal metastases. Ann Surg. 2002;235(6):759-66.

9. Wei AC, Greig PD, Grant D, et al. Survival after hepatic resection for colorectal metastases: a 10-year experience. Ann Surg Oncol. 2006;13(5):668-76.

10. Andreou A, Aloia TA, Brouquet A, et al. Margin status remains an important determinant of survival after surgical resection of colorectal liver metastases in the era of modern chemotherapy. Ann Surg. 2013;257(6):1079-88.

11. Cunningham D, Pyrhonen S, James RD, et al. Randomised trial of irinotecan plus supportive care versus supportive care alone after fluorouracil failure for patients with metastatic colorectal cancer. Lancet. 1998;352(9138):1413-8.

12. de Gramont A, Figer A, Seymour M, et al. Leucovorin and fluorouracil with or without oxaliplatin as first-line treatment in advanced colorectal cancer. J Clin Oncol. 2000;18(16):2938-47.

13. Saltz LB, Clarke S, Diaz-Rubio E, et al. Bevacizumab in combination with oxaliplatin based chemotherapy as first-line therapy in metastatic colorectal cancer: a randomized phase III study. J Clin Oncol. 2008;26(13):2013-9.

14. Van Cutsem E, Kohne CH, Hitre E, et al. Cetuximab and chemotherapy as initial treatment for metastatic colorectal cancer. N Engl J Med. 2009;360(14):1408-17.

15. Bokemeyer C, Bondarenko I, Makhson A, et al. Fluorouracil, leucovorin, and oxaliplatin with and without cetuximab in the first-line treatment of metastatic colorectal cancer. J Clin Oncol. 2009;27(5):663-71.

16. Van Cutsem E, Rivera F, Berry S, et al. Safety and efficacy of first-line bevacizumab with folfox, xelox, folfiri and fluoropyrimidines in metastatic colorectal cancer: the BEAT study. Ann Oncol. 2009;20(11):1842-7.
17. Moertel CG, Fleming TR, MacDonald JS, et al. Levamisole and fluorouracil for adjuvant therapy of resected colon carcinoma. N Engl J Med. 1990;322(6):352-8.

18. • Portier G, Elias D, Bouche O, et al. Multicenter randomized trial of adjuvant fluouracil and folinic acid compared with surgery alone after resection of colorectal liver metastases: FFCD ACHBTH AURC 9002 trial. J Clin Oncol. 2006;24(31):4976-82. Prospective randomized clinical trial comparing hepatic metastasectomy to hepatic metastasectomy plus adjuvant 5FU/leucovorin chemotherapy.

19. •• Mitry E, Fields AL, Bleiberg H, et al. Adjuvant chemotherapy after potentially curative resection of metastases from colorectal cancer: a pooled analysis of two randomized trials. J Clin Oncol. 2008;26(30):4906-11. Pooled analyses of two prospective randomized clinical trials comparing hepatic metastasectomy to hepatic metastasectomy plus adjuvant 5FU/leucovorin chemotherapy.

20. Ychou M, Hohenberger W, Thezenas S, et al. A randomized phase III study comparing adjuvant 5-fluorouracil/folinic acid with FOLFIRI in patients following complete resection of liver metastases from colorectal cancer. Ann Oncol. 2009;20(12):1964-70.

21. • Nordlinger B, Sorbye H, Glimelius B, et al. Perioperative chemotherapy with FOLFOX4 and surgery versus surgery alone for resectable liver metastases from colorectal cancer (EORTC Intergroup trial 40983): a randomised controlled trial. Lancet. 2008;371(9617):1007-16. Prospective randomized clinical trial comparing hepatic metastasectomy to hepatic metastasectomy plus perioperative FOLFOX6 chemotherapy.

22. •• Nordlinger B, Sorbye H, Glimelius B, et al. Perioperative FOLFOX chemotherapy and surgery versus surgery alone for resectable liver metastases from colorectal cancer (EORTC 40983): long-term results of a randomised, controlled, phase 3 trial. Lancet Oncol. 2013;14(12):1208-15. Longer-term follow-up of reference 21.

23. Cho CS. The oncological significance of postoperative complications after hepatic colorectal metastasectomy: biology, technique, or statistical quirk? J Surg Res. 2012;172(1):80-2.

24. Andtbacka RH, Ng CS, Scaife CL, et al. Surgical resection of gastrointestinal stromal tumors after treatment with imatinib. Ann Surg Oncol. 2007;14(1):14-24.

25. Rutkowski P, Gronchi A, Hohenberger P, et al. Neoadjuvant imatinib in locally advanced gastrointestinal stromal tumors (GIST): the EORTC STBSG experience. Ann Surg Oncol. 2013;20(9):2937-43.

26. - Vauthey JN, Pawlik TM, RIbero D, et al. Chemotherapy regimen predicts steatohepatitis and an increase in 90-day mortality after surgery for hepatic colorectal metastases. J Clin Oncol. 2006;24(13):2065-72. Analysis of correlation between chemotherapeutic regimen and both hepatic steatohepatitis and postoperative complications.

27. Fernandez FG, Ritter J, Goodwin JW, et al. Effect of steatohepatitis associated with irinotecan or oxaliplatin pretreatment on resectability of hepatic colorectal metastases. J Am Coll Surg. 2005;200(6):845-53.

28. Kooby DA, Fong Y, Suriawinata A, et al. Impact of steatosis on perioperative outcome following hepatic resection. J Gastrointest Surg. 2003;7(8):1034-44.

29. Aloia T, Sebagh M, Plasse M, et al. Liver histology and surgical outcomes after preoperative chemotherapy with fluorouracil plus oxaliplatin in colorectal cancer liver metastases. J Clin Oncol. 2006;24:4983-90.

30. Rubbia-Brandt L, Audard V, Sartoretti P, et al. Severe hepatic sinusoidal obstruction associated with oxaliplatin-based chemotherapy in patients with metastatic colorectal cancer. Ann Oncol. 2004;15:460-6.

31. Karoui M, Penna C, Amin-Hashem M, et al. Influence of preoperative chemotherapy on the risk of major hepatectomy for colorectal liver metastases. Ann Surg. 2006;243:1-7. 
32. Vigano L, Capussotti L, De Rosa G, et al. Liver resection for colorectal metastases after chemotherapy: impact of chemotherapy-related liver injuries, pathological tumor response, and micrometastases on long-term survival. Ann Surg. 2013;258:731-40.

33. Cho CS, Curran S, Schwartz LH, et al. Preoperative radiographic assessment of hepatic steatosis with histologic correlation. J Am Coll Surg. 2008;206(3):480-8.

34. Kapiteijn E, Marijnen CA, Nagtegaal ID, et al. Preoperative radiotherapy combined with total mesorectal excision for resectable rectal cancer. N Engl J Med. 2001;345(9):638-46.

35. Suh KW, Kim BW, Chun M, Lim HY. Is postoperative radiotherapy useful for the rectal carcinoma in the era of total mesorectal excision? Hepatogastroenterology. 2002;49(44):399-403.

36. MacDonald JS, Smalley SR, Benedetti J, et al. Chemoradiotherapy after surgery versus surgery alone for adenocarcinoma of the stomach or gastroesophageal junction. $\mathrm{N}$ Engl $\mathrm{J}$ Med. 2001;345(10):725-30.

37. Cunningham D, Allum WH, Stenning SP, et al. Perioperative chemotherapy versus surgery alone for gastroesophageal cancer. N Engl J Med. 2006;355(1):11-20.

38. Correa-Gallego C, Gonen M, Fischer M, et al. Perioperative complications influence recurrence and survival after resection of hepatic colorectal metatases. Ann Surg Oncol. 2013;20(8):2477-84.

39. Aloia TA, Fahy BN, Fischer CP, et al. Predicting poor outcome following hepatectomy: analysis of 2,313 hepatectomies in the NSQIP database. HPB (Oxford). 2009;11(6):510-5.

40. Breslin TM, Hess KR, Harbison DB, et al. Neoadjuvant chemoradiotherapy for adenocarcinoma of the pancreas: treatment variables and survival duration. Ann Surg Oncol. 2001;8(2):123-32.

41. Allen PJ, Kemeny N, Jarnagin WR, et al. Importance of response to neoadjuvant chemotherapy in patients undergoing resection of synchronous colorectal liver metastases. J Gastrointest Surg. 2003;7(1):109-15.

42. Blazer DG 3rd, Kishi Y, Maru DM, et al. Pathologic response to preoperative chemotherapy: a new outcome end point after resection of hepatic colorectal metastases. J Clin Oncol. 2008;26(33):5344-51.
43. Gallagher DJ, Zheng J, Capanu M, et al. Response to neoadjuvant chemotherapy does not predict overall survival for patients with synchronous hepatic metastases. Ann Surg Oncol. 2009;16(7): 1844-51.

44. Cho CS, Labow DM, Tang L, et al. Histologic grade is correlated with outcome after resection of hepatic neuroendocrine neoplasms. Cancer. 2008;113(1):126-34.

45. DeMatteo RP, Ballman KV, Antonescu CR, et al. Adjuvant imatinib mesylate after resection of localised, primary gastrointestinal stromal tumor: a randomised, double-blind, placebocontrolled trial. Lancet. 2009;373(9669):1097-104.

46. Ambiru S, Miyazaki M, Isono T, et al. Hepatic resection for colorectal metastases: analysis of prognostic factors. Dis Colon Rectum. 1999;42(5):632-9.

47. Donato N, Dario C, Giovanni S, et al. Retrospective study on adjuvant chemotherapy after surgical resection of colorectal cancer metastatic to the liver. Eur J Surg Oncol. 1994;20(4): 454-60.

48. Kemeny N, Huang Y, Cohen AM, et al. Hepatic arterial infusion of chemotherapy after resection of hepatic metastases from colorectal cancer. N Engl J Med. 1999;341(27):2039-48.

49. - Andreou A, Kopetz S, Maru DM, et al. Adjuvant chemotherapy with FOLFOX for primary colorectal cancer is associated with increased somatic gene mutations and inferior survival in patients undergoing hepatectomy for metachronous liver metastases. Ann Surg. 2012;256(4):642-50. Retrospective analysis suggesting that the use of systemic chemotherapy for colorectal adenocarcinoma may promote the selection of a more aggressive phenotype of metastatic disease.

50. - Parks R, Gonen M, Kemeny N, et al. Adjuvant chemotherapy improves survival after resection of hepatic colorectal metastases: analysis of data from two continents. J Am Coll Surg. 2007;204(5):753-61. Retrospective analysis suggesting that the survival benefit associated with adjuvant chemotherapy after hepatic metastasectomy may be more pronounced for patients with a greater burden of metastatic disease. 